\title{
Determination of inhibitor activity of drugs against the COVID-19
}

\author{
Gedikli MA ${ }^{1}$, Tuzun $\mathrm{B}^{2}$, Sayin $\mathrm{K}^{2}$, Ataseven $\mathrm{H}^{3}$ \\ Department of Internal Medicine, Faculty of Medicine, Sivas Cumhuriyet University, Sivas, Turkey. \\ krysayin@gmail.com
}

\section{ABSTRACT}

BACKGROUND: It is the SARS-CoV-2 virus, one of the most significant diseases of today's world. Due to the high transmission of this disease, studies are ongoing to discover an inhibitor drug that can stop this disease. In this study, inhibitory drugs used for many diseases were tried to stop the SARS-CoV-2 virus.

AIM: In the calculations made, inhibitor molecules for the SARS-CoV-2 virus were calculated by molecular docking method.

RESULTS AND CONCLUSION: Inhibitory activities of SARS-CoV-2 virus against spike glycoprotein (PDB ID: $6 \mathrm{MOJ}, 6 \mathrm{LZG}$ ), main protease (PDB ID: 5RGG, 6WTT), and RNA dependent RNA polymerase (RdRp) (PDB ID: 6YYT, 7BV2) proteins were compared. Then, docking calculations were supported by calculations by MM-PSBA of the inhibitor with the highest activity. Afterwards, it was compared with FDA approved drugs for the SARS-CoV-2 virus. It was found that the Carvedilol molecule was the best against RNA dependent RNA polymerase (RdRp) protein of SARS-CoV-2 (Tab. 4, Fig. 9, Ref. 42). Text in PDF www.elis.sk KEY WORDS: SARS-CoV-2, molecular docking, COVID-19, ADME/T, inhibitory activities.

\section{Introduction}

In December 2019, a new coronavirus agent was identified as the cause of this disease, which started with respiratory system symptoms such as fever, shortness of breath, and cough in the $\mathrm{Hu}-$ bei province of China and spread rapidly all over the world. The World Health Organization (WHO) named the virus that causes this disease called COVID-19, severe acute respiratory syndrome coronavirus 2 (SARS-CoV-2) (1). Due to the rapid spread of the virus worldwide and its high infectivity, WHO recognized this disease as a pandemic in March 2020. From the beginning of the COVID-19 outbreak until September 2020, it has been reported that more than 25 million people were infected with this virus and more than 850 thousand people died.

Coronaviruses were first identified in patients with flu symptoms in 1966 by Tyrell and Bynoe (2). It is the seventh subtype of

\footnotetext{
${ }^{1}$ Department of Internal Medicine, Faculty of Medicine, Sivas Cumhuriyet University, Sivas, Turkey, ${ }^{2}$ Department of Biochemistry, Faculty of Science, Sivas Cumhuriyet University, Sivas, Turkey, and ${ }^{3}$ Department of Gastroenterology, Faculty of Medicine, Sivas Cumhuriyet University, Sivas, Turkey
}

Address for correspondence: Koray Sayin, Ass Prof Dr, Sivas Cumhuriyet University, Faculty of Science, Department of Chemistry, 58140 Sivas, Turkey.

Acknowledgments: This study is supported by the Scientific Research Project Fund of Sivas Cumhuriyet University under project number RGD020. This study was made possible by TUBITAK ULAKBIM, High Performance, and Grid Computing Center (TR-Grid e-Infrastructure). the coronavirus family, which has been shown to cause disease with the newly emerged SARS-CoV-2 virus. When the genomic structure of the coronavirus that causes COVID-19 is examined, it is seen that it is in the same subgenus with the severe acute respiratory syndrome (SARS) but in a different class (3). Coronaviruses can cause disease in both humans and animals. Especially alpha and beta coronaviruses are caused by bats. SARS-CoV-2's genomic structure is highly similar to bat coronavirus (4).

Coronaviruses consist of four structural proteins: S (Spike) protein, $\mathrm{E}$ (Envelope) protein, $\mathrm{M}$ (Membrane) protein, and $\mathrm{N}(\mathrm{Nu}-$ cleocapsid) protein. The $\mathrm{S}$ protein is responsible for the formation of spikes on the surface of the virus. These protrusions are responsible for both the attachment of the virus to the host cell receptors and the entry of the virus into the cell. Because of this feature, the $\mathrm{S}$ protein constitutes the prominent antigenic feature of the virus. The $\mathrm{N}$ protein contains regions in the RNA structure that bind to the viral genome. $\mathrm{N}$ protein binds with nsp3 (nonstructural protein 3 ), allowing the genome to bind to the replication-transcription complex. The E protein plays a role in the packaging and release of the virus. With the M protein, the virus binds to the nucleocapsid (5). SARS COV-2 enters the cell by binding to the angiotensinconverting enzyme 2 (ACE2) receptor via the S protein. Also, another way that is thought to be essential for the entry of the virus into the cell is the cellular protease TMPRSS2 (6).

Although the mode of transmission of SARS-CoV-2 from person to person is not absolute, it is thought to occur by respiratory droplets. The virus released through droplets during coughing and sneezing of COVID-19 positive individuals can be transmitted to another person by direct contact (7). 


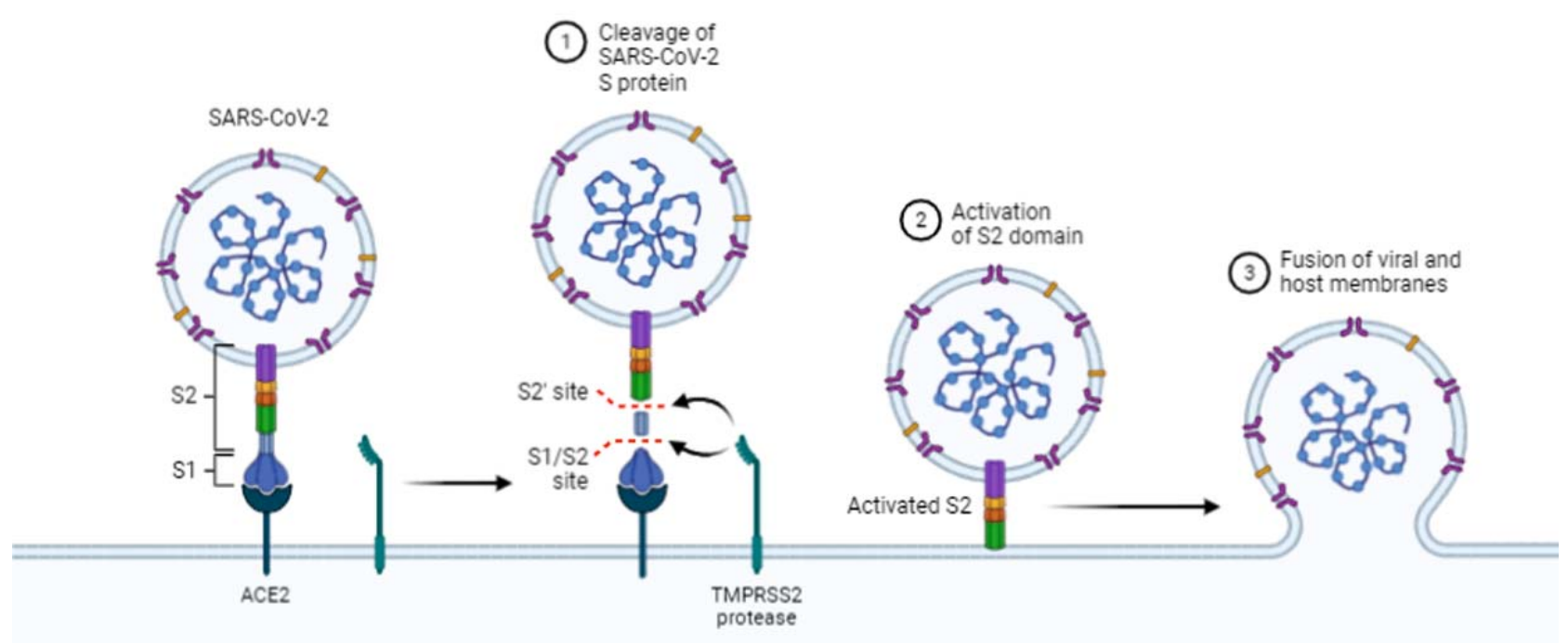

Target cell

Fig. 1. Illustration of the SARS-CoV-2 virus entry into the cell.

Although there is no laboratory finding specific to COVID-19 disease, lymphopenia, thrombocytopenia, increased CRP, prolonged prothrombin time (PT), acute kidney damage, high Ddimer, troponin elevation, $\mathrm{LDH}$, and ferritin elevation indicate that the disease will progress severely (9). The current diagnostic method of COVID-19 is based on detecting the virus by polymerase chain reaction (PCR). There may be false negativities caused by incorrect sample taking or testing. In order to prevent false negativities, determining antibodies against the virus, together with the PCR test, will enable the virus to be detected more accurately $(10,11)$.

To date, no specific anti-viral therapy or vaccine has been found that has proven effective against COVID-19. Many drugs such as dexamethasone, hydroxychloroquine/chloroquine, remdesivir, favipiravir, azithromycin, tocilizumab, lopinavir-ritonavir are used in combination or alone depending on the severity of the disease in patients infected with COVID-19 (12-14).

The experimental studies performed show that when the SARS-CoV-2 virus penetration into the cell in human metabolism was examined in detail, it was seen that the SARS-CoV-2 virus attaches to the ACE2 proteins. The entry of the SARS-CoV-2 virus into the cell is shown in detail in Figure 1. An inhibitor is used to prevent the SARS-CoV-2 virus from entering the cell. In this study, the activities of drugs approved by the U.S. Food and Drug Administration (FDA) and active ingredients of medicinal herbs against the SARS-CoV-2 virus are compared. For this purpose, 179 inhibitors were scanned, and their molecular structures were downloaded from the PubChem site (https://pubchem.ncbi. nlm.nih.gov/). As proteins of the SARS-CoV-2 virus used in this study; spike glycoprotein (PDB ID: 6M0J (15), 6LZG (16)), main protease (PDB ID: 5RGG, 6WTT (17)), and RNA dependent RNA polymerase (RdRp) (PDB ID: 6YYT (18), 7BV2 (19)) of the SARS-CoV-2 virus proteins. The molecular mechanics Poisson-
Boltzmann surface binding free energy values of area (MM-PBSA) calculations study interactions between inhibitor molecules and SARS-CoV-2 virus proteins at 100000 ps. Afterward, ADME/T analysis of the molecules with the highest inhibitory activity among these 179 FDA-approved drugs and active ingredients of medicinal herbs molecules was performed.

\section{Method}

In this study, molecular docking calculations were made to compare the inhibitory activities of one hundred eighty-one molecules against the proteins of the SARS-CoV-2 virus. With these calculations, many parameters about molecules have been obtained. The numerical values of these parameters obtained as a result of the calculations provide essential information about the inhibitory activities of the molecules against the proteins of the SARS-CoV-2 virus.

Molecular docking calculations made to compare the inhibitory activities of molecules against the SARS-CoV-2 virus were calculated using the Maestro Molecular modeling platform (version 12.2) by the Schrödinger program (20). For these calculations, proteins and molecules of the SARS-CoV-2 virus must be prepared. Each protein and molecule made was calculated separately. Different operations were carried out in each calculation phase. First, it was used from the Gaussian software program (21) to obtain optimized structures of inhibitor molecules, by using these structures *.sdf extension files were prepared. Using these files, all calculations were made with the Maestro Molecular modeling platform (version 12.2) by Schrödinger, LLC that comes together from many modules. In the first module of the calculations, the protein preparation module $(22,23)$ is used to prepare proteins for calculations. For calculations, the active sites of the enzymes were determined, in which freedom of movement was given to 

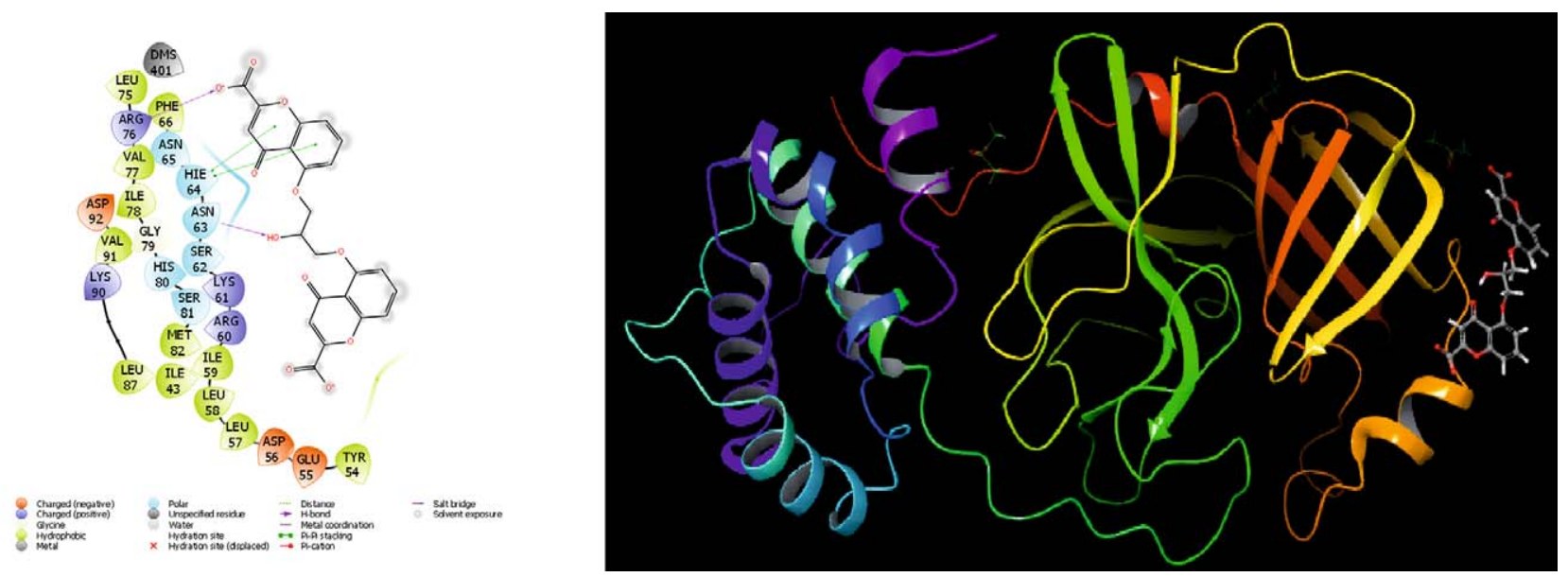

Fig. 2. Interaction of molecule 2882 with 5 RGG protein of SARS-CoV-2 virus.

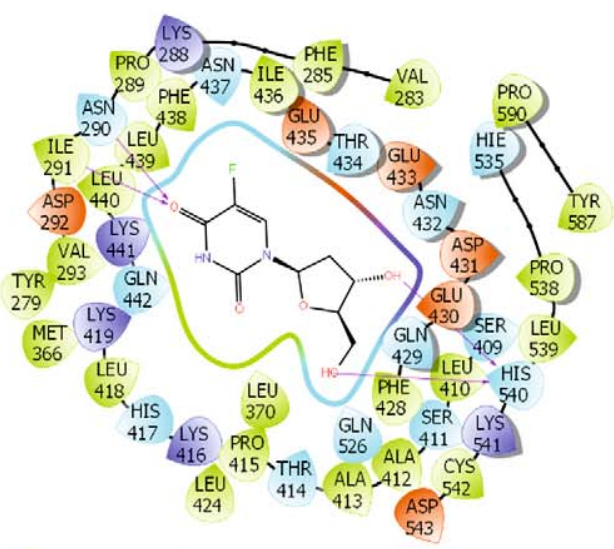

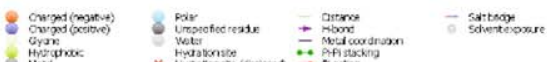

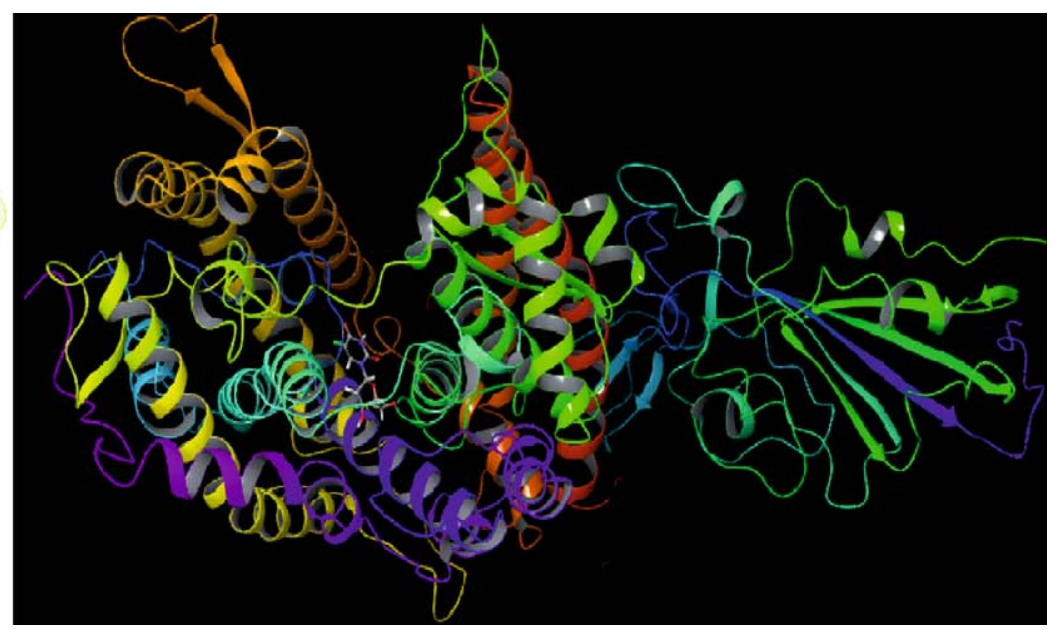

Fig. 3. Interaction of molecule 5790 with 6LZG protein of SARS-CoV-2 virus
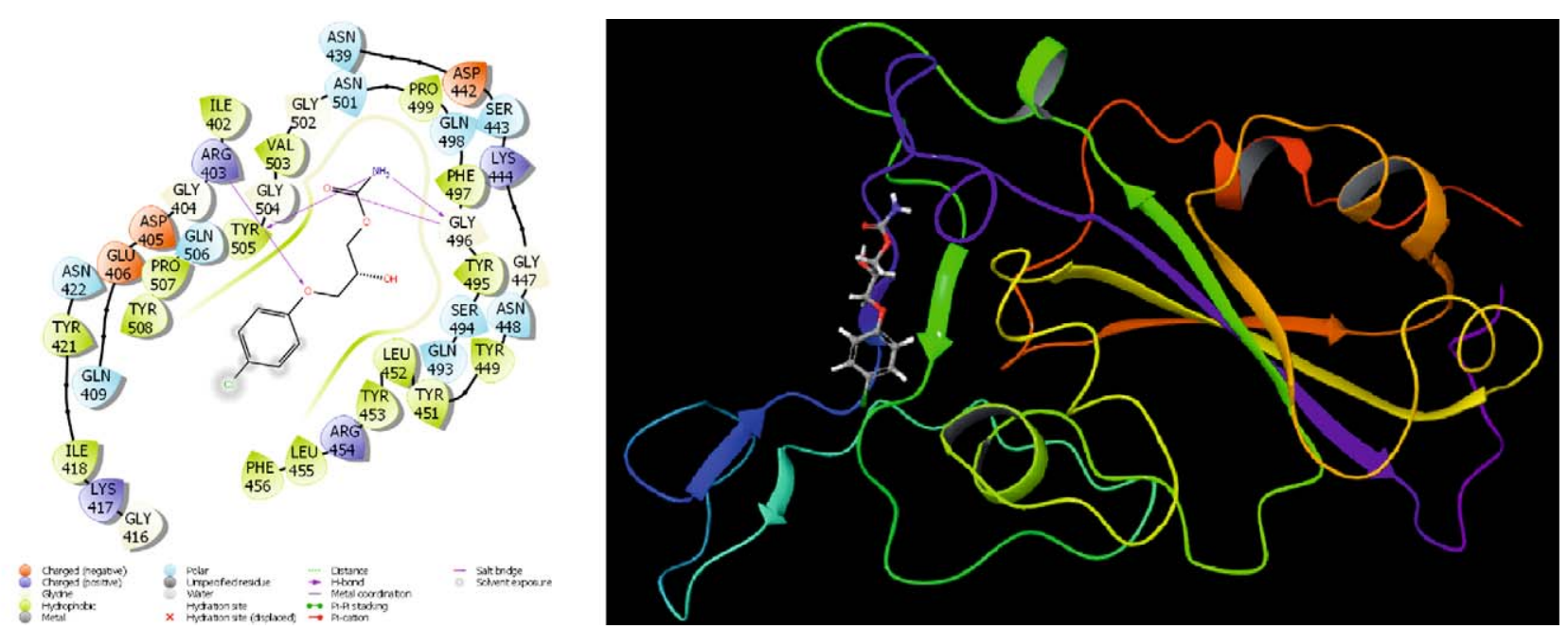

Fig. 4. Interaction of molecule 2724 with $6 \mathrm{MOJ}-$ protein of SARS-CoV-2 virus. 
497-506
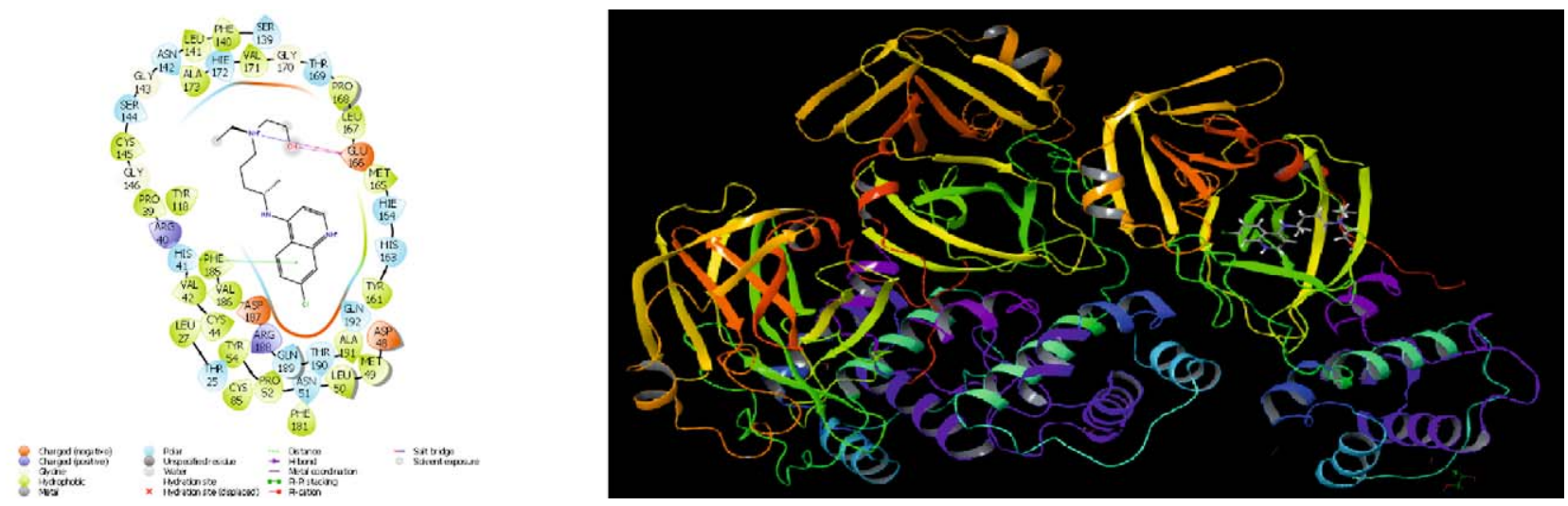

Fig. 5. Interaction of molecule 3652 with $6 \mathrm{WTT}$ protein of SARS-CoV-2 virus.
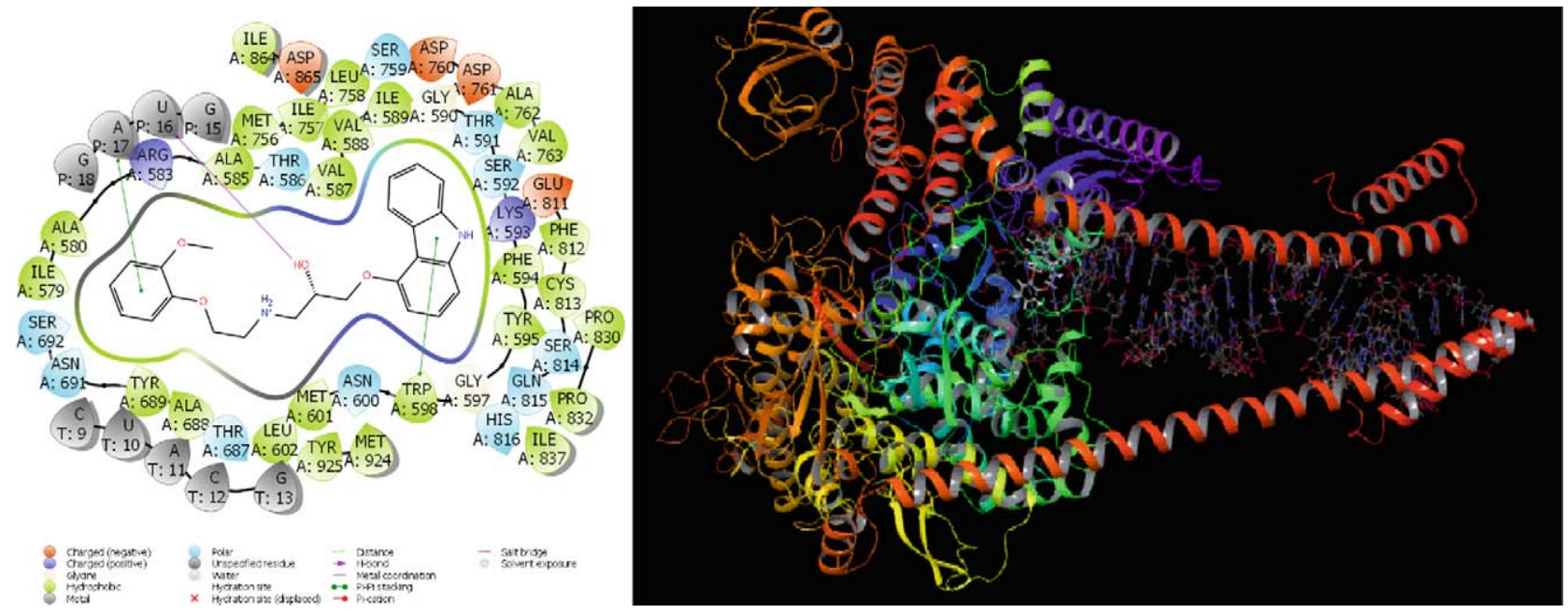

Fig. 6. Interaction of molecule 2585 of 6yyt-with protein of SARS-CoV-2 virus.
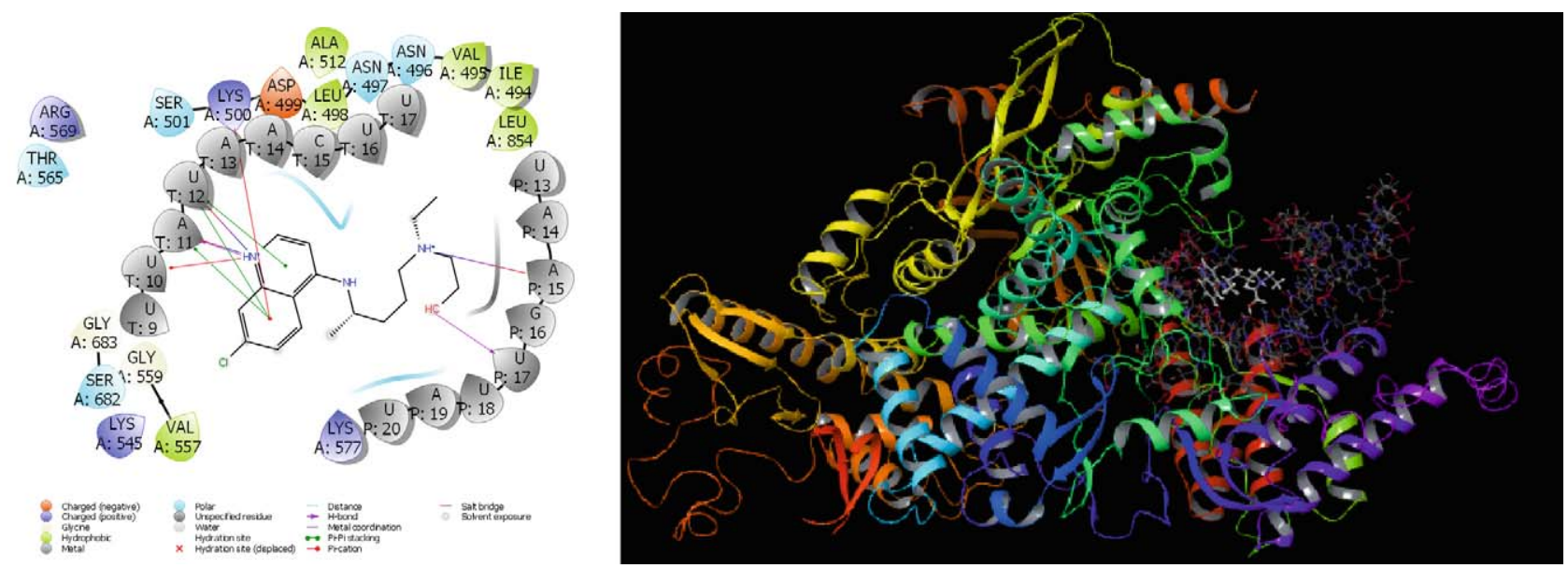

Fig. 7. Interaction of molecule 3652 with $7 \mathrm{bv} 2$ protein of SARS-CoV-2 virus. 
Tab. 1. Numerical values of the docking parameters of inhibitor molecule against SARSCoV-2 proteins.

\begin{tabular}{|c|c|c|c|c|c|c|c|}
\hline & $\begin{array}{l}\text { Docking } \\
\text { score }\end{array}$ & $\begin{array}{l}\text { Glide ligand } \\
\text { efficiency }\end{array}$ & $\begin{array}{l}\text { Glide } \\
\text { ecoul }\end{array}$ & $\begin{array}{l}\text { Glide } \\
\text { emodel }\end{array}$ & $\begin{array}{l}\text { Glide } \\
\text { energy }\end{array}$ & $\begin{array}{c}\text { Glide } \\
\text { einternal }\end{array}$ & $\begin{array}{c}\text { Glide } \\
\text { posenum }\end{array}$ \\
\hline \multicolumn{8}{|l|}{$6 \mathrm{M} 0 \mathrm{~J}$} \\
\hline 2724 & -4.07 & -0.25 & -13.57 & -6.73 & -32.28 & -20.30 & 2.12 \\
\hline 2585 & -3.97 & -0.13 & -5.28 & -12.40 & -19.24 & -17.69 & 0.95 \\
\hline 2882 & -3.81 & -0.11 & -26.16 & -12.84 & -47.14 & -39.00 & 15.90 \\
\hline 3415 & -3.71 & -0.53 & -9.59 & -13.78 & -28.23 & -23.37 & 1.27 \\
\hline 3862 & -3.62 & -0.26 & -28.44 & -4.42 & -45.67 & -32.87 & 5.63 \\
\hline 3314 & -3.27 & -0.27 & -5.44 & -7.48 & -14.77 & -12.92 & 2.97 \\
\hline 3598 & -3.15 & -0.15 & -21.11 & -6.15 & -37.86 & -27.26 & 3.23 \\
\hline \multicolumn{8}{|l|}{ 7BV2 } \\
\hline 3652 & -8.82 & -0.38 & -27.87 & -27.81 & -73.24 & -55.67 & 11.04 \\
\hline 2585 & -8.18 & -0.27 & -27.82 & -29.24 & -73.69 & -57.06 & 5.84 \\
\hline 2165 & -7.38 & -0.30 & -29.49 & -18.74 & -64.21 & -48.24 & 13.78 \\
\hline 2719 & -7.07 & -0.32 & -27.78 & -30.28 & -79.48 & -58.06 & 3.16 \\
\hline 2724 & -5.27 & -0.33 & -36.04 & -20.64 & -77.21 & -56.68 & 7.60 \\
\hline 3955 & -5.07 & -0.15 & -15.72 & -10.94 & -30.90 & -26.66 & 1.58 \\
\hline \multicolumn{8}{|l|}{ 6WTT } \\
\hline 3652 & -6.37 & -0.28 & -27.98 & -12.56 & -50.07 & -40.54 & 3.51 \\
\hline 2882 & -5.80 & -0.17 & -42.73 & -5.37 & -61.31 & -48.10 & 2.80 \\
\hline 2165 & -5.58 & -0.22 & -34.35 & -8.60 & -50.29 & -42.96 & 7.57 \\
\hline 2724 & -5.44 & -0.34 & -30.11 & -4.31 & -42.67 & -34.42 & 1.95 \\
\hline 3314 & -5.00 & -0.42 & -18.81 & -4.65 & -27.33 & -23.45 & 0.54 \\
\hline \multicolumn{8}{|l|}{ 5RGG } \\
\hline 2882 & -4.48 & -0.13 & -24.34 & -11.31 & -43.64 & -35.64 & 8.94 \\
\hline 2585 & -4.46 & -0.15 & -2.47 & -12.45 & -15.68 & -14.92 & 0.13 \\
\hline 2165 & -3.75 & -0.15 & -21.23 & -13.48 & -41.45 & -34.72 & 5.61 \\
\hline 3415 & -3.49 & -0.50 & -25.79 & -6.52 & -37.87 & -32.30 & 1.63 \\
\hline 3652 & -3.33 & -0.14 & -16.04 & -6.15 & -23.24 & -22.19 & 1.65 \\
\hline 2724 & -3.26 & -0.20 & -7.60 & -10.57 & -18.20 & -18.17 & 2.99 \\
\hline 2536 & -3.23 & -0.11 & -11.69 & -4.48 & -15.66 & -16.17 & 0.99 \\
\hline 2092 & -3.20 & -0.11 & -23.32 & -12.40 & -41.70 & -35.72 & 7.68 \\
\hline \multicolumn{8}{|l|}{$6 \mathrm{YYT}$} \\
\hline 2585 & -8.96 & -0.30 & -2.76 & -22.09 & 13.69 & -24.86 & 14.85 \\
\hline 5790 & -8.43 & -0.50 & -18.10 & -30.17 & -80.19 & -48.27 & 7.49 \\
\hline 3652 & -8.18 & -0.36 & -8.25 & -24.97 & -50.14 & -33.22 & 5.19 \\
\hline 2719 & -7.68 & -0.35 & -28.71 & -15.46 & -58.64 & -44.17 & 1.29 \\
\hline 5789 & -7.58 & -0.45 & -32.95 & -13.25 & -53.32 & -46.20 & 4.55 \\
\hline 4631 & -7.07 & -0.37 & -23.48 & -15.26 & -49.48 & -38.74 & 7.43 \\
\hline 3862 & -7.03 & -0.50 & -27.07 & -11.34 & -50.31 & -38.41 & 1.06 \\
\hline \multicolumn{8}{|l|}{ 6LGZ } \\
\hline 5790 & -8.33 & -0.49 & -25.45 & -9.48 & -42.63 & -34.93 & 0.00 \\
\hline 2585 & -8.11 & -0.27 & -37.52 & -17.9 & -80.79 & -55.42 & 6.12 \\
\hline 5789 & -7.71 & -0.45 & -27.36 & -11.9 & -47.37 & -39.25 & 5.76 \\
\hline 5475 & -7.16 & -0.24 & -33.78 & -9.58 & -59.39 & -43.36 & 8.85 \\
\hline 2882 & -6.93 & -0.2 & -38.51 & -8.00 & -64.51 & -46.51 & 4.16 \\
\hline 2724 & -6.76 & -0.42 & -31.81 & -10.77 & -55.61 & -42.59 & 4.13 \\
\hline 5625 & -6.68 & -0.21 & -50.56 & -3.09 & -85.6 & -53.65 & 6.98 \\
\hline 4631 & -6.53 & -0.34 & -28.09 & -10.37 & -49.37 & -38.46 & 8.53 \\
\hline
\end{tabular}

of molecular docking calculations using this module are used. After the docking calculations, ADME/T analysis (absorption, distribution, metabolism, excretion, and toxicity) was performed to examine the future drug properties of the molecule. The Qik-prop module (27) of the Schrödinger software was used for ADME/T analysis.

Finally, molecular mechanics Poisson-Boltzmann surface area (MM-PB$\mathrm{SA})$ calculations of the interaction between the molecule 2585 and 6YYT that is the RNA dependent RNA polymerase protein of the SARS-CoV-2 virus, was calculated. In these calculations, numerical values were calculated by using $\mathrm{Na}$ noscale Molecular Dynamics (NAMD) (28) and Visual Molecular Dynamics (VMD) (29) software programs. These numerical values calculated are the numerical values of many chemical interactions between inhibitor molecules and proteins. These chemical interaction values are binding free energy, van der Waals energy, electrostatic energy, kinetic energy, and potential energy changes. Binding-free energy value provides information about biochemical interactions between inhibitors and proteins. The Binding free energy value between protein and inhibitor consists of many energy values such as potential energy, polar and non-polar solvation energies (30). In the calculations made, free binding energy value and total free energy value of inhibitor, protein, and inhibitor-protein are calculated separately.

$$
\Delta G_{\text {Binding }}=G_{\text {Complex }}-\left(G_{\text {Protein }}+G_{\text {Inhibitor }}\right)
$$

In this equation, $\Delta G_{\text {Binding }}$ is the difference between the binding free energy value inhibitor and protein, $G_{\text {Complex }}, G_{\text {Protein }}$ and $G_{\text {Inhibitor }}$ are values of total free energy of protein-ligand complex, protein and inhibitor respectively (31). Each term in the equation given above consists of the sum of many energy values. These ener-

all proteins in this active site. Therefore, these proteins have been enabled to interact more easily with molecules. In the next step, the LigPrep module $(24,25)$ was used in preparation for the calculations of the working molecules.

In the next step, the prepared protein and molecules were docked with each other. The Glide ligand docking module (26) was used for this step. In this module, the OPLS3e method was used in all calculations for docking calculations of molecules and proteins. Numerical values of many parameters obtained as a result gies are van der Waals energy, electrostatic energy, and polar contribution.

\section{Result and discussion}

Inhibitory activities of FDA approved drugs, and active ingredients of medicinal herbs against the SARS-CoV-2 virus were investigated. The numerical values of the inhibitory activities of FDA approved drugs and medicinal herbs against the SARS-CoV-2 
Tab. 2. ADME properties of molecules.

\begin{tabular}{|c|c|c|c|c|c|c|}
\hline & 2585 & 2724 & 2882 & 3652 & $5790 x$ & Referance range \\
\hline mol_MW & 406 & 246 & 468 & 336 & 246 & $130-725$ \\
\hline dipole (D) & 2.1 & 3.9 & 10.6 & 8.3 & 8.2 & $1.0-12.5$ \\
\hline SASA & 766 & 502 & 752 & 639 & 414 & $300-1000$ \\
\hline FOSA & 250 & 85 & 72 & 325 & 125 & $0-750$ \\
\hline FISA & 72 & 184 & 349 & 50 & 222 & $7-330$ \\
\hline PISA & 443 & 161 & 332 & 193 & 22 & $0-450$ \\
\hline WPSA & 0.0 & 71.5 & 0.0 & 71.5 & 45.3 & $0-175$ \\
\hline volume $\left(\mathrm{A}^{3}\right)$ & 1341 & 794 & 1325 & 1129 & 694 & $500-2000$ \\
\hline donorHB & 3.0 & 3.0 & 3.0 & 2.0 & 3.0 & $0-6$ \\
\hline accptHB & 5.5 & 5.0 & 12.2 & 5.7 & 8.6 & $2.0-20.0$ \\
\hline glob $($ Sphere $=1)$ & 0.8 & 0.8 & 0.8 & 0.8 & 0.9 & $0.75-0.95$ \\
\hline QPpolrz $\left(\mathrm{A}^{3}\right)$ & 44.7 & 23.3 & 44.3 & 34.4 & 19.9 & $13.0-70.0$ \\
\hline QPlogPC16 & 15.2 & 8.9 & 16.1 & 11.4 & 7.0 & $4.0-18.0$ \\
\hline QPlogPoct & 21.8 & 15.0 & 27.7 & 17.7 & 17.0 & $8.0-35.0$ \\
\hline $\mathrm{QP} \log \mathrm{Pw}$ & 12.4 & 11.2 & 20.6 & 9.3 & 14.6 & $4.0-45.0$ \\
\hline $\mathrm{QP} \log \mathrm{Po} / \mathrm{w}$ & 4.2 & 1.0 & 1.9 & 3.5 & -1.0 & $-2.0-6.5$ \\
\hline $\mathrm{QP} \log \mathrm{S}$ & -4.9 & -2.9 & -4.2 & -3.3 & -1.7 & $-6.5-0.5$ \\
\hline CIQPlogS & -5.2 & -2.6 & -5.0 & -3.3 & -1.6 & $-6.5-0.5$ \\
\hline QPlogHERG & -8.1 & -5.1 & -3.1 & -6.1 & -3.0 & $*$ \\
\hline QPPCaco (nm/sec) & 512 & 179 & 0 & 826 & 77 & $* *$ \\
\hline QPlogBB & -0.5 & -1.4 & -3.8 & 0.0 & -1.3 & $-3.0-1.2$ \\
\hline QPPMDCK $(\mathrm{nm} / \mathrm{sec})$ & 265 & 190 & 0 & 1096 & 55 & $* *$ \\
\hline QPlogKp & -2.3 & -3.8 & -5.9 & -2.9 & -5.2 & $\mathrm{Kp}$ in $\mathrm{cm} / \mathrm{hr}$ \\
\hline $\mathrm{IP}(\mathrm{ev})$ & 8.0 & 9.2 & 9.4 & 8.1 & 9.7 & $7.9-10.5$ \\
\hline $\mathrm{EA}(\mathrm{eV})$ & 0.1 & 0.1 & 1.3 & 0.6 & 0.7 & $-0.9-1.7$ \\
\hline \#metab & 5.0 & 3.0 & 3.0 & 5.0 & 3.0 & $1-8$ \\
\hline QPlogKhsa & 0.6 & -0.5 & -0.8 & 0.1 & -0.8 & $-1.5-1.5$ \\
\hline Human Oral Absorption & 3.0 & 3.0 & 1.0 & 3.0 & 2.0 & - \\
\hline Per. Human Oral Absorp. & 100 & 73 & 16 & 100 & 55 & $* * *$ \\
\hline PSA & 70 & 95 & 206 & 47 & 127 & $7-200$ \\
\hline RuleOfFive & 0.0 & 0.0 & 1.0 & 0.0 & 0.0 & Maximum is 4 \\
\hline RuleOfThree & 0.0 & 0.0 & 1.0 & 0.0 & 0.0 & Maximum is 3 \\
\hline $\mathrm{Jm}$ & 0.0 & 0.0 & 0.0 & 0.2 & 0.0 & - \\
\hline
\end{tabular}

virus were compared (32). It should be well known that the most important factor affecting this numerical value is the interaction between molecules and proteins. It has been observed that as this interaction increases, the inhibitory activities of the molecules increase. Because there are many interactions between molecules and proteins. Increasing these interactions will increase the binding of the inhibitor to the protein. These interactions have many interactions, such as hydrogen bonds, polar and hydrophobic interactions, $\pi-\pi$, and halogen (33-39). The demonstrations of interactions between inhibitors and proteins of the SARS-CoV-2 virus are shown in Figures 2-6.

In this study, in which FDA approved drugs and active ingredients of medicinal herbs were used as inhibitors, many parameters were obtained by molecular docking calculations to compare the inhibitory activities of inhibitor molecules against proteins. With the numerical values of these calculated parameters, important information about the activities of the inhibitor molecules is obtained. Among these calculated parameters, the most important parameter is the docking score parameter of the inhibitor molecules. The inhibitor whose numerical value is the most positive for this parameter is worse than the others. In the light of the above information, when it is desired to compare the inhibitory activities of inhibitor molecules, the most negative molecule inhibitory activity of the docking score parameter of the inhibitor molecules is the highest $(40,41)$.

Many parameters other than the docking score parameter were calculated from their interactions with inhibitor molecules against the SARS-CoV-2 virus. Many of these parameters are Glide hbond, Glide evdw, and Glide ecoul parameters used to explain what chemical interactions occur during this interaction, of which only Glide ecoul was calculated (40). In this parameter, the numerical value of the Coulomb interactions that occur between the inhibitors and the proteins of the SARS-CoV-2 virus is calculated. This interaction is given in Figures 2-7.

Apart from these parameters, Glide emodel, Glide energy, Glide internal, and Glide posenum parameters give numerical data about the interaction pattern between SARS-CoV-2 virus proteins and inhibitor molecules. Each parameter gives different information. These parameters provide numerical information about the posse formed as a result of interaction between inhibitor molecules and proteins of the SARS-CoV-2 virus. The numerical values of the parameters obtained from the interaction of the inhibitor molecules with the proteins of the SARS-CoV-2 virus are shown in (Tab. 1).

As a result of the calculations, considering the obtained docking score parameter, chlorphenesin carbamate molecule is the best inhibitor against the spike protein of SARS-CoV-2 virus with 2724 PubChem ID with a numerical value of -4.07 . Hydroxychloroquine molecule is the best inhibitor of SARS-CoV-2 virus against RNA dependent RNA polymerase ( $\mathrm{RdRp}$ ) protein with PubChem ID 3652 with a numerical value of -8.82 . Hydroxychloroquine molecule is the best inhibitor against the main protease protein of the SARS-CoV-2 virus with a PubChem ID of -6.37 with a numerical value of 3652 . Carvedilol molecule is the best inhibitor against RNA dependent RNA polymerase (RdRp) protein of SARS-CoV-2 virus with PubChem ID of 2585 with a numerical value of -8.96 . With PubChem ID 5790 with a numerical value -8.33 of, the Carvedilol molecule is the best inhibitor against the spike protein of the SARS-CoV-2 virus.

Considering the values given in Table 2, ADME/T analysis of the molecules with the highest docking score parameters was performed. In this analysis, the movements of the inhibitor mole- 
Tab. 3. Representation of calculated parameters (kcal/mol) and standard deviation values of 6YYT protein and molecule 2585.

\begin{tabular}{ccccccccc}
\hline TIME $(\mathrm{ps})$ & \multicolumn{2}{c}{ VDW } & \multicolumn{2}{c}{ KINETIC } & \multicolumn{2}{c}{ POTENTIAL } & \multicolumn{2}{c}{ Gibbs binding free energy } \\
\hline 5000 & -33883.60 & \pm 4000.5 & -89520.67 & \pm 9923.3 & 414349.46 & \pm 13888.3 & -324442.17 & \pm 97129.0 \\
\hline 10000 & -32112.48 & \pm 594.2 & -91626.71 & \pm 670.6 & 410785.98 & \pm 841.6 & -318751.49 & \pm 1364.7 \\
\hline 15000 & -32239.84 & \pm 334.1 & -92098.10 & \pm 106.7 & 410929.32 & \pm 22.0 & -318452.00 & \pm 121.5 \\
\hline 20000 & -32320.14 & \pm 559.6 & -91740.83 & \pm 369.7 & 410779.40 & \pm 409.8 & -318629.45 & \pm 422.1 \\
\hline 25000 & -31954.93 & \pm 53.4 & -91429.05 & \pm 244.4 & 412125.89 & \pm 832.8 & -320310.38 & \pm 1090.0 \\
\hline 30000 & -32876.87 & \pm 440.5 & -91533.33 & \pm 74.8 & 412086.36 & \pm 162.4 & -320163.72 & \pm 86.3 \\
\hline 35000 & -32322.38 & \pm 226.9 & -91492.36 & \pm 151.3 & 411132.27 & \pm 162.4 & -319250.65 & \pm 310.6 \\
\hline 40000 & -31604.29 & \pm 654.8 & -91379.52 & \pm 68.5 & 410246.67 & \pm 325.1 & -318459.28 & \pm 266.2 \\
\hline 45000 & -33023.53 & \pm 515.0 & -91793.30 & \pm 38.5 & 411242.08 & \pm 249.4 & -319067.12 & \pm 217.0 \\
\hline 50000 & -32047.71 & \pm 718.1 & -91504.41 & \pm 259.9 & 411385.78 & \pm 217.8 & -319481.64 & \pm 45.2 \\
\hline 55000 & -32740.40 & \pm 39.2 & -91913.20 & \pm 402.2 & 412440.01 & \pm 456.9 & -320149.48 & \pm 53.1 \\
\hline 60000 & -31984.56 & \pm 291.8 & -91782.16 & \pm 137.7 & 411706.38 & \pm 101.0 & -319537.12 & \pm 234.8 \\
\hline 65000 & -32845.16 & \pm 220.4 & -91580.77 & \pm 154.4 & 410901.85 & \pm 311.9 & -318932.35 & \pm 163.8 \\
\hline 70000 & -32223.71 & \pm 87.3 & -92111.21 & \pm 224.9 & 411374.05 & \pm 101.5 & -318849.26 & \pm 121.9 \\
\hline 75000 & -3222.25 & \pm 515.3 & -92081.79 & \pm 37.2 & 411743.80 & \pm 105.5 & -319264.38 & \pm 136.8 \\
\hline 80000 & -31469.62 & \pm 425.1 & -91879.41 & \pm 34.6 & 411364.72 & \pm 27.7 & -319074.10 & \pm 2.7 \\
\hline 85000 & -31698.88 & \pm 135.9 & -92928.18 & \pm 634.4 & 411240.50 & \pm 3.5 & -317898.24 & \pm 651.4 \\
\hline 90000 & -32933.98 & \pm 135.6 & -91840.36 & \pm 504.8 & 411787.81 & \pm 290.8 & -319548.16 & \pm 797.5 \\
\hline 100000 & -32770.86 & \pm 226.6 & -92269.35 & \pm 31.7 & 413145.14 & \pm 703.9 & -320474.85 & \pm 661.7 \\
\hline
\end{tabular}

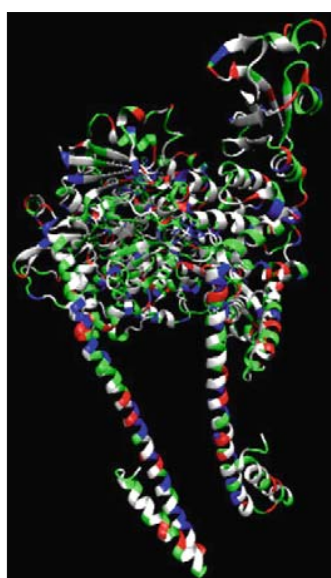

$0 \mathrm{~ns}$

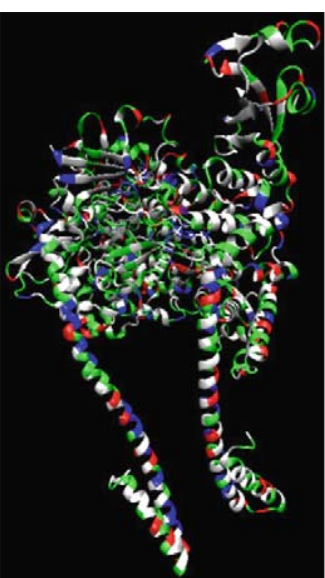

$25 \mathrm{~ns}$

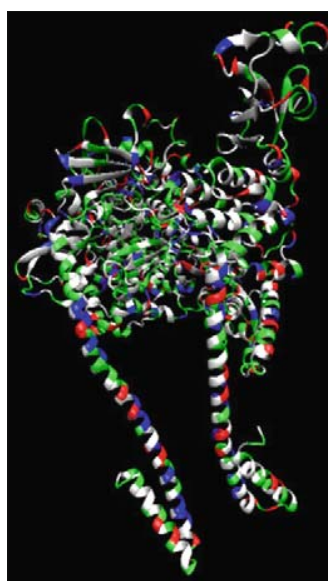

$50 \mathrm{~ns}$

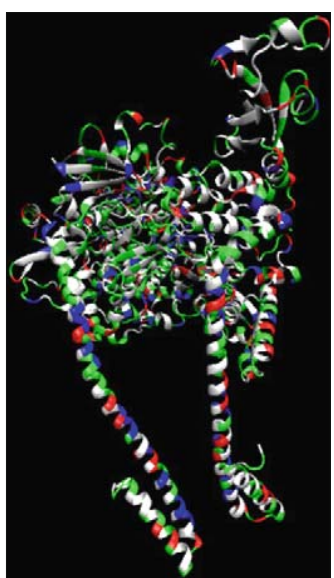

$75 \mathrm{~ns}$

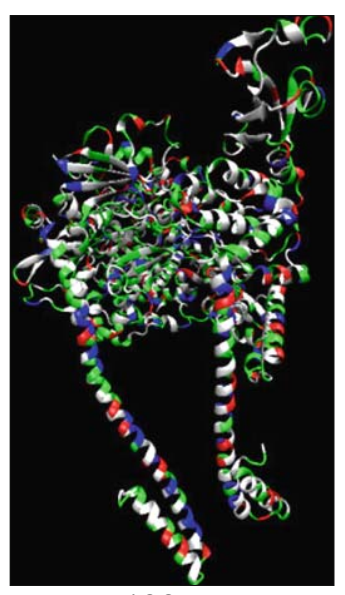

$100 \mathrm{~ns}$

Fig. 8. Representation of the interaction between molecule 2585 and 6YYT protein of the SARS-CoV-2 virus in the $25 \mathrm{~ns}$ range.

cules in human metabolism were tried to be predicted. Since the way in which inhibitory molecules should be taken into the human body, it tries to predict theoretically which reactions will be given in which organ. Many parameters were calculated for these estimates and predictions. Among these parameters, the mol MW parameter gives information about the molar mass of the inhibitor molecules. Another parameter, donorHB parameter, shows the capacity of molecules to form hydrogen bonds. Another parameter is the parameter QPPCaco, which is the numerical value of the permeability of Caco-2 cells. It is known that Caco-2 cells reside in the gut and that Caco-2 cells represent the gut-blood barrier. Another parameter is QPPMDCK, which is a numerical value of cell permeability for MDCK, MDCK is the blood-brain barrier in the brain. The next parameter is Per. It is Human Oral Absorp., which gives the oral absorption value of the molecules as a result of the oral use of the molecules. The next parameter is RuleOfFive (40), which does not consist of five rules, even though it contains five words. This rule is also known as Pfizer's rule of five. These parameter rules are mol_MW $<500, \mathrm{QP} \log \mathrm{Po} / \mathrm{w}<5$, donorHB $\leq$ 5 , accptHB $\leq 10$. On the other hand, the RuleOfThree parameter (41) is known as Jorgensen's rule of three. The three rules are: QP $\log \mathrm{S}>-5.7$, QP PCaco > $22 \mathrm{~nm} / \mathrm{s}$, \# Primary Metabolites $<7$. Each rule that complies with these rules (RuleOfFive and RuleOfThree) counts as one. If the rules that do not follow reach the number of rules, this drug molecule cannot be used as a drug. These rules prefer zero as a number.

Finally, against SARS-CoV-2 proteins, among the molecules that are expected to be used as drugs, the 2585 molecule, which 
497-506

Tab. 4. Numerical values of the docking parameters of molecule against enzymes.

\begin{tabular}{lccccccccc}
\hline Protein & Inhibitor & Docking score & $\begin{array}{c}\text { Glide ligand } \\
\text { efficiency }\end{array}$ & Glide evdw & Glide ecoul & Glide energy & Glide einternal & Glide emodel & Glide hBond \\
\hline 6M0J & 37542 & -4.50 & -0.26 & -19.74 & -5.20 & -24.95 & 3.48 & -37.19 & -1.88 \\
& 84029 & -2.57 & -0.05 & -20.46 & -7.48 & -27.94 & 15.61 & 10000 & -1.18 \\
& 92727 & -2.50 & -0.05 & -33.47 & -11.57 & -45.04 & 12.39 & -58.92 & -1.46 \\
& 131411 & -1.38 & -0.05 & -27.55 & -2.58 & -30.12 & 3.80 & -42.67 & 0.00 \\
\hline 5RGG & 37542 & -4.26 & -0.25 & -12.66 & -9.40 & -22.06 & 4.27 & -26.29 & -2.68 \\
& 121304016 & -3.19 & -0.08 & -37.43 & -11.24 & -48.66 & 9.35 & -53.75 & -1.63 \\
& 492405 & -3.05 & -0.28 & -10.30 & -7.91 & -18.21 & 0.01 & -21.00 & -1.31 \\
\hline 7BV2 & 37542 & -6.94 & -0.41 & -15.81 & -23.93 & -39.74 & 3.44 & -50.88 & -4.38 \\
\hline
\end{tabular}

has the highest biological activity, has a numerical value of -8.96 against the 6YYT protein, the RNA-dependent RNA polymerase protein of the SARS-CoV-2 virus. The interaction of this molecule with the RNA dependent RNA polymerase protein has been studied in detail by calculations of MM-PBSA. Only molecular docking calculations have some disadvantages. Because there is no stability in the molecular docking nanosecond unit. Although protein is rigid and inhibitors are more flexible in docking calculations, both are flexible in MM-PBSA calculations.

In MM-PBSA calculations, protein and inhibitor molecules are flexible and surrounded by solvent molecules. In these calculations, the interaction between protein and inhibitor molecules was examined at intervals of 100 picoseconds. Gibbs free energy values between protein and inhibitor are calculated for every 100 picoseconds and are given in Table 3. At 0 picosecond, Gibbs free energy is at the lowest level. This value gradually increases for every next 100 picoseconds to be around the value of -320000 $\mathrm{kcal} / \mathrm{mol}$. The VDW, kinetic, potential, and Gibbs binding free energy values calculated from the interaction between the 6YYT protein of the SARS-CoV-2 virus and molecule 2585 are given in Table 3. With these values obtained by the molecular mechanics-Poisson-Boltzmann surface area (MMPBSA) method, it has been tried to support the docking results. The negative numerical value of VDW, KINETIC, and Gibbs binding free energy values indicates better binding of protein and inhibitor molecules to each other (42). The interaction between molecule 2585 and 6 YYT protein of the SARS-CoV-2 virus is shown in Figure 8 at 25 ns intervals.

In order to find an ideal drug molecule with the numerical value of the parameters obtained as a result of docking calculations, it is necessary to compare it with the existing drug molecules. These drug molecules are found in U.S. Pat. There are drugs approved by the Food and Drug Administration (FDA). These drugs can be used against viruses and bacteria in various diseases. The FDA office, which is affiliated with the United States Department of Health, is the bureau responsible for food, dietary supplements, pharmaceuticals, biological medical products, blood products, medical devices, radiation devices, veterinary instruments, and cosmetics. Especially drugs used against the SARS-CoV-2 virus are FDA approved. By comparing the inhibitor molecules studied with these drugs, more effective and more active inhibitors were tried to be found.
In order to find an ideal drug molecule with the numerical value of the parameters obtained as a result of docking calculations, it is necessary to compare it with the existing drug molecules. These drug molecules are drugs approved by the U.S. Food and Drug Administration (FDA) in the United States. These drugs can be used against viruses and bacteria in various diseases. The FDA office, which is affiliated with the United States Department of Health, is the bureau responsible for food, dietary supplements, pharmaceuticals, biological medical products, blood products, medical devices, radiation devices, veterinary instruments, and cosmetics. Especially drugs used against the SARS-CoV-2 virus are FDA approved. By comparing these drugs with inhibitor molecules studied, more effective and more active inhibitors have been found. The numerical values obtained are given in Table 4 .

These drugs are ribavirin (PubChem number: 37542), arbidol (PubChem number: 131411), favipiravir (PubChem number: 492405), remdesivir (PubChem number: 121304016), clarithromycin (PubChem number: 84029), lopinavir (PubChem number: 92727), and azithromycin (PubChem number: 447043). These drugs are FDA approved drugs used for different proteins of the SARS-CoV-2 virus in different countries, ribavirin drug is one of the most effective drugs for different proteins. It is an FDA approved ribavirin drug against the 6M0J protein of the SARS$\mathrm{CoV}-2$ virus. Although the docking score parameter for this protein is -4.50 , the docking score parameter of the inhibitors numbered
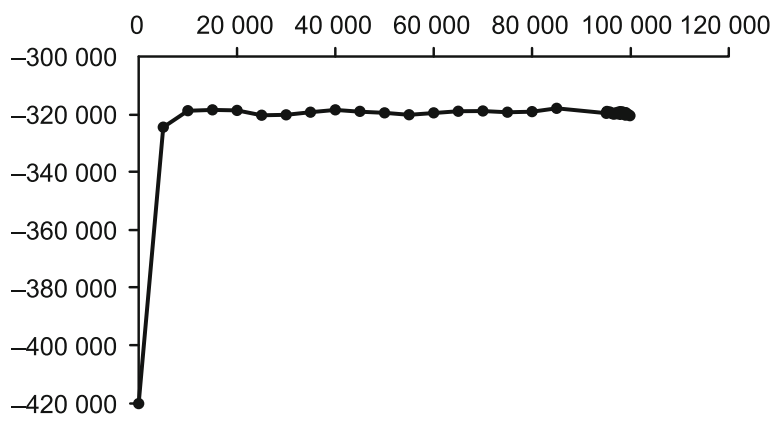

Fig. 9. Exchange of Gibbs binding free energy values in the interaction between molecule 2585 and 6 YYT protein of the SARS-CoV-2 virus. 
2724 in PubChem against this protein is -4.07 . The docking score parameter of the FDA approved drug against the 5RGG protein of another SARS-CoV-2 virus is -4.26 , whereas the docking score parameter of the inhibitors numbered 2882 in PubChem against this protein is -4.48 . In the last protein, although the docking score parameter of the FDA approved drug against the 7BV2 protein of the SARS-CoV-2 virus is -6.94 , the docking score parameter of inhibitors numbered 3652 in PubChem is -8.82 against this protein. Calculations show that the inhibitors numbered 3652 and 2882 PubChem are better than the FDA approved drugs.

\section{Conclusion}

As a result of the calculations made, the inhibitory activities of the inhibitor molecules of drugs used for various diseases against the SARS-CoV-2 virus were compared. When the numerical values of the obtained parameters are examined, it is seen that the inhibitors with the number 3652 and 2882 PubChem are more effective than the FDA approved drugs. Finally, the interaction of 2585 PubChem numbered inhibitor with the highest inhibitory activity against SARS-CoV-2 protein was supported by calculations of MM-PBSA, and docking calculations were supported. These theoretical calculations will guide further in vivo and in vitro experimental studies.

\section{References}

1. Velavan TP, Meyer CG. The COVID-19 epidemic. Trop Med Internat Health 2020; 25 (3): 278.

2. Tyrrell D, Bynoe M. Cultivation of viruses from a high proportion of patients with colds. Lancet 1966: 76-77.

3. Zhu N, Zhang D, Wang W, Li X, Yang B, Song J et al. A novel coronavirus from patients with pneumonia in China, 2019. New Engl J Med 2020.

4. Zhou P, Yang X-L, Wang X-G, Hu B, Zhang L, Zhang W et al. A pneumonia outbreak associated with a new coronavirus of probable bat origin. Nature 2020; 579 (7798): 270-273.

5. Chen Y, Liu Q, Guo D. Emerging coronaviruses: genome structure, replication, and pathogenesis. J Med Virol 2020; 92 (4): 418-423.

6. Hoffmann M, Kleine-Weber H, Krüger N, Mueller MA, Drosten C, Pöhlmann S. The novel coronavirus 2019 (2019-nCoV) uses the SARScoronavirus receptor ACE2 and the cellular protease TMPRSS2 for entry into target cells. BioRxiv 2020.

7. Wu D, Wu T, Liu Q, Yang Z. The SARS-CoV-2 outbreak: what we know. Internat J Infect Dis 2020.

8. Li Q, Guan X, Wu P, Wang X, Zhou L, Tong Y et al. Early transmission dynamics in Wuhan, China, of novel coronavirus-infected pneumonia. New Engl J Med 2020.

9. Liao D, Zhou F, Luo L, Xu M, Wang H, Xia J et al. Haematological characteristics and risk factors in the classification and prognosis evaluation of COVID-19: a retrospective cohort study. Lancet Haematol 2020 .

10. Rothe C, Schunk M, Sothmann P, Bretzel G, Froeschl G, Wallrauch C et al. Transmission of 2019-nCoV infection from an asymptomatic contact in Germany. New Engl J Med 2020; 382 (10): 970-971.
11. Corman VM, Landt O, Kaiser M, Molenkamp R, Meijer A, Chu DK et al. Detection of 2019 novel coronavirus (2019-nCoV) by real-time RT-PCR. Eurosurveillance 2020; 25 (3): 2000045.

12. Aktaş A, Tüzün B, Aslan R, Sayin K, Ataseven H. New anti-viral drugs for the treatment of COVID-19 instead of favipiravir. J Biomol Struct Dynam 2020: 1-11.

13. Siemieniuk RA, Bartoszko JJ, Ge L, Zeraatkar D, Izcovich A, Pardo-Hernandez H et al. Drug treatments for covid-19: living systematic review and network meta-analysis. BMJ 2020; 370.

14. Wang M, Cao R, Zhang L, Yang X, Liu J, Xu M et al. Remdesivir and chloroquine effectively inhibit the recently emerged novel coronavirus (2019-nCoV) in vitro. Cell Res 2020; 30 (3): 269-271.

15. Lan J, Ge J, Yu J, Shan S, Zhou H, Fan S et al. Structure of the SARS-CoV-2 spike receptor-binding domain bound to the ACE2 receptor. Nature 2020; 581 (7807): 215-220.

16. Wang Q, Zhang Y, Wu L, Niu S, Song $C$, Zhang $Z$ et al. Structural and functional basis of SARS-CoV-2 entry by using human ACE2. Cell 2020.

17. Ma C, Sacco MD, Hurst B, Townsend JA, Hu Y, Szeto T et al. Boceprevir, GC-376, and calpain inhibitors II, XII inhibit SARS-CoV-2 viral replication by targeting the viral main protease. bioRxiv 2020 .

18. Hillen HS, Kokic G, Farnung L, Dienemann C, Tegunov D, Cramer P. Structure of replicating SARS-CoV-2 polymerase. bioRxiv 2020.

19. Yin W, Mao C, Luan X, Shen D-D, Shen Q, Su H et al. Structural basis for inhibition of the RNA-dependent RNA polymerase from SARSCoV-2 by remdesivir. Science 2020.

20. Schrodinger L. Small-Molecule Drug Discovery Suite 2019-4 2019. New York, NY: Schrodinger, LLC 2019.

21. Frisch M, Trucks G, Schlegel HB, Scuseria GE, Robb MA, Cheeseman JR et al. gaussian 09, Revision d. 01, Gaussian. Inc, Wallingford CT 2009; 201.

22. Epik SL. New York NY, 2019-4. Impact, Schrödinger, LLC, New York, NY 2016; Protein Preparation Wizard.

23. Friesner RA, Murphy RB, Repasky MP, Frye LL, Greenwood JR, Halgren TA et al. Extra precision glide: Docking and scoring incorporating a model of hydrophobic enclosure for protein- ligand complexes. J Med Chem 2006; 49 (21): 6177-6196.

24. Sastry GM, Adzhigirey M, Day T, Annabhimoju R, Sherman W. Protein and ligand preparation: parameters, protocols, and influence on virtual screening enrichments. J Comp Mol Design 2013; 27 (3): 221-234.

25. Release S. LigPrep, Schrödinger, LLC, New York, NY, 2019-4. New York, NY 2019.

26. Du Q, Qian Y, Yao X, Xue W. Elucidating the tight-binding mechanism of two oral anticoagulants to factor Xa by using induced-fit docking and molecular dynamics simulation. J Biomol Struct Dynam 2019.

27. Release S. QikProp, Schrödinger, LLC, New York, NY, 2019-4. New York, NY 2019.

28. Nelson MT, Humphrey W, Gursoy A, Dalke A, Kalé LV, Skeel RD et al. NAMD: a parallel, object-oriented molecular dynamics program. Internat J Supercomp Appl High Performance Computing 1996; 10 (4): 251-268.

29. Humphrey W, Dalke A, Schulten K. VMD: visual molecular dynamics. J Mol Graph 1996; 14 (1): 33-38. 


\section{7-506}

30. Abdelrheem DA, Abd El-Mageed H, Mohamed HS, Rahman AA, Elsayed KN, Ahmed SA. Bis-indole alkaloid caulerpin from a new source Sargassum platycarpum: isolation, characterization, in vitro anticancer activity, binding with nucleobases by DFT calculations and MD simulation. J Biomol Struct Dynam 2020: 1-11.

31. Koçyiğit ÜM, Taslimi P, Tüzün B, Yakan H, Muğlu H, Güzel E. 1, 2, 3-Triazole substituted phthalocyanine metal complexes as potential inhibitors for anticholinesterase and antidiabetic enzymes with molecular docking studies. J Biomol Struct Dynam 2020: 1-11.

32. Aktas A, Tuzun B, Taskin A, Sayin K, Ataseven H. How do arbidol and its analogs inhibit the SARS-CoV-2? Bratisl Med J 2020; 121 (10): 705-711.

33. Sayin K, Karakaş D. Determination of structural, spectral, electronic and biological properties of tosufloxacin boron complexes and investigation of substituent effect. J Mol Struct 2017; 1146: 191-197.

34. Sayin K, Karakaş D. Investigation of structural, electronic properties and docking calculations of some boron complexes with norfloxacin: A computational research. Spectrochimica Acta Part A: Mol Biomol Spectroscop 2018; 202: 276-283.

35. Sayin K, Karakaș D. Quantum chemical investigation of levofloxacinboron complexes: a computational approach. J Mol Struct 2018; 1158: $57-65$.

36. Sayin K, Üngördü A. Investigation of anticancer properties of caffeinated complexes via computational chemistry methods. Spectrochimica Acta Part A: Mol Biomol Spectroscop 2018; 193: 147-155.
37. Sayin K, Üngördü A. Investigations of structural, spectral and electronic properties of enrofloxacin and boron complexes via quantum chemical calculation and molecular docking. Spectrochimica Acta Part A: Mol Biomol Spectroscop 2019; 220: 117102.

38. Üngördü A, Sayin K. Quantum chemical calculations on sparfloxacin and boron complexes. Cheml Phys Lett 2019; 733: 136677.

39. Jayarajan R, Satheeshkumar R, Kottha T, Subbaramanian S, Sayin K, Vasuki G. Water mediated synthesis of 6-amino-5-cyano-2-oxo-N- (pyridin-2-yl)-4- (p-tolyl)-2H-[1, 2'-bipyridine]-3-carboxamide and 6-amino5-cyano-4- (4-fluorophenyl)-2-oxo-N- (pyridin-2-yl)-2H-[1, 2'-bipyridine]3-carboxamide-An experimental and computational studies with non-linear optical (NLO) and molecular docking analyses. Spectrochimica Acta Part A: Mol Biomol Spectroscop 2020; 229: 117861.

40. Celebioglu HU, Erden Y, Hamurcu F, Taslimi P, Şentürk OS, Özmen ÜÖ et al. Cytotoxic effects, carbonic anhydrase isoenzymes, $\alpha$-glycosidase and acetylcholinesterase inhibitory properties, and molecular docking studies of heteroatom-containing sulfonyl hydrazone derivatives. J Biomol Struct Dynam 2020: 1-12.

41. Huseynova A, Kaya R, Taslimi P, Farzaliyev V, Mammadyarova $\mathbf{X}$, Sujayev A et al. Design, synthesis, characterization, biological evaluation, and molecular docking studies of novel 1,2-aminopropanthiols substituted derivatives as selective carbonic anhydrase, acetylcholinesterase and $\alpha$-glycosidase enzymes inhibitors. J Biomol Struct Dynam 2020: 1-13.

42. Gupta MK, Vemula S, Donde R, Gouda G, Behera L, Vadde R. In-silico approaches to detect inhibitors of the human severe acute respiratory syndrome coronavirus envelope protein ion channel. J Biomol Struct Dynam 2020: 1-11. 